\title{
Extensive chaos in Rayleigh-Bénard convection
}

\author{
M. R. Paul* and M. I. Einarsson \\ Department of Mechanical Engineering, Virginia Polytechnic and State University, Blacksburg, Virginia 24061, USA \\ P. F. Fischer \\ Mathematics and Computer Science Division, Argonne National Laboratory, Argonne, Illinois 60439, USA \\ M. C. Cross \\ Department of Physics, California Institute of Technology, Pasadena, California 91101, USA
}

(Received 15 August 2006; published 26 April 2007)

\begin{abstract}
Using large-scale numerical calculations we explore spatiotemporal chaos in Rayleigh-Bénard convection for experimentally relevant conditions. We calculate the spectrum of Lyapunov exponents and the Lyapunov dimension describing the chaotic dynamics of the convective fluid layer at constant thermal driving over a range of finite system sizes. Our results reveal that the dynamics of fluid convection is truly chaotic for experimental conditions as illustrated by a positive leading-order Lyapunov exponent. We also find the chaos to be extensive over the range of finite-sized systems investigated as indicated by a linear scaling between the Lyapunov dimension of the chaotic attractor and the system size.
\end{abstract}

DOI: 10.1103/PhysRevE.75.045203

PACS number(s): 05.45.Jn, 05.10.-a, 47.20.Bp, 47.52.+j

Many open challenges in science and engineering are due to the complex dynamics of spatially extended systems that are driven far from equilibrium [1]. Examples include the weather and climate, the trajectories of hurricanes, earthquakes, the patterns of growing colonies of microorganisms, manufacturing uniform materials from a melt, and the convection of suspended organisms in the oceans and rivers. These systems form intricate spatial patterns that affect the transport of mass, momentum, and energy, which then affect the spatial patterns. These interactions are often nonlinear and present a significant obstacle to furthering our understanding of many real-world systems. A common feature of spatially extended nonequilibrium systems is spatiotemporal chaos, where the dynamics are aperiodic in time and space (aperiodic time dynamics alone is often referred to as chaos). A great deal of progress has been made in understanding spatiotemporal chaos from studies of simplified models of spatially extended systems. However, it remains unclear whether these insights apply to experimentally accessible systems. In this Rapid Communication we present largescale calculations that give insight into the spatiotemporal chaos of an experimentally accessible system. Computations such as these provide a quantitative link between theoretical ideas and the dynamics of spatiotemporal chaos for realworld systems.

A particular open challenge is to understand the origins and structure of the active degrees of freedom of complex dynamics in extended systems. However, in most physical systems the complex dynamics is the result of many competing factors including strong external driving (such as fluid turbulence), many interacting components (such as the complex regulatory networks common in biology), or large system size (such as the weather and climate). Of these possi-

\footnotetext{
*Electronic address: mrp@vt.edu; URL: http://www.me.vt.edu/ $\sim$ mpaul
}

bilities the case of large system size is particularly promising in light of the modern supercomputers and improved parallel algorithms that are now available. In what follows we explore numerically the development of spatiotemporal chaos in a fluid system for fixed driving as a function of increasing system size for the precise conditions of experiment.

Much of our current understanding of chaos has come from studies where the chaotic attractor of the system dynamics can be described geometrically in terms of only a few chaotic degrees of freedom. However, it is not clear how to proceed when the dimension of the attractor becomes large as is expected for most experimentally relevant systems [2-6]. In this case geometrical descriptions of the attractor become very difficult and prohibitive to implement [5]. Many significant open questions remain. For example, of the infinite degrees of freedom in a continuous system (actually several million degrees of freedom on a computer) how many are excited? What are the origin, structure, and dynamics of the chaotic degrees of freedom? How do these chaotic degrees of freedom enter the dynamics as the system size is increased? Are there important features of the striking visual patterns of many nonequilibrium systems that significantly contribute to spatiotemporal chaos?

It is possible to begin to address these questions by appealing to the defining feature of chaos: the exponential separation of trajectories of solutions in phase space from solutions that originate from nearly identical initial conditions [5]. The separation in phase space is quantified by the spectrum of Lyapunov exponents $\lambda_{i}$ where $i=1, \ldots, N$ with $\lambda_{i}$ arranged in descending order. The leading-order exponent $\lambda_{1}$ describes the growth of the line separating two trajectories in phase space, $\lambda_{1}+\lambda_{2}$ describes the growth of a twodimensional area of initial conditions, and $\sum_{i=1}^{N} \lambda_{i}$ describes the growth of an $\mathrm{N}$-dimensional ball of initial conditions. For many practical systems there will be a finite number of exponents that yield a positive result when added together. The exact number of exponents required for the sum to vanish corresponds to the dimension of the ball of initial conditions 
that will neither grow nor shrink under the dynamics (often called the Lyapunov dimension $D_{\lambda}$ ). Given only the Lyapunov exponents, $D_{\lambda}$ can be determined from the Kaplan-Yorke formula $D_{\lambda}=k+S_{k} /\left|\lambda_{k+1}\right|$, where $k$ is the largest $n$ for which $S_{n}=\sum_{i=1}^{n} \lambda_{i}>0$ [6]. The value of $D_{\lambda}$ is the minimum number of active degrees of freedom that contribute to the chaotic dynamics $[7,8]$. The Lyapunov exponents are extremely difficult to measure experimentally since it is usually not possible to begin experiments from slightly different initial conditions and the perturbations quickly grow beyond the linear regime as required by the linearization in the definition of the Lyapunov exponents. In addition, the Lyapunov exponents are also computationally intensive to calculate since an additional linearized solution must be calculated simultaneously for each desired exponent. However, with the advent of large supercomputers and improved numerical algorithms these calculations are now possible for experimentally accessible systems as we show here.

Ruelle [9] was the first to conjecture that for very large systems the Lyapunov dimension should scale extensively with the size of the system, $D_{\lambda} \propto \Gamma^{d_{s}}$, where $\Gamma$ is the system size and $d_{s}$ is the number of spatially extended dimensions. Extensive chaos has been confirmed in a variety of simple model systems [10-15] and for experimentally motivated systems with nonphysical boundary conditions $[2,3]$. However, these arguments have not been tested for experimentally realistic systems.

We study the spatiotemporal chaos of Rayleigh-Bénard convection (RBC) given by the buoyancy-driven convection of a thin layer of fluid heated uniformly from below. RBC is a canonical pattern-forming system in which theoretical and experimental research continues to provide important new insights into the dynamics of nonequilibrium systems. Governing the fluid motion of Rayleigh-Bénard convection are the well-known Boussinesq equations, a set of nonlinear partial differential equations which yield the fluid velocity, pressure, and temperature as a function of time [1]. The control parameter $R$, where $R$ is the Rayleigh number, is proportional to the constant temperature difference across the fluid layer and is the key parameter that is varied during a typical convection experiment; small values of $R$ correspond to simple, often time-independent, flows; intermediate values of $R$ correspond to complex chaotic flows as studied here (see Fig. 1 ); and very large values of $R$ correspond to strongly driven turbulent flow. It is now possible to solve these equations numerically for convection domains with the precise conditions of experiment using a geometrically flexible and highly efficient, parallel, spectral element method [16] (for its use with RBC see, for example, [17]). In our simulations we impose the no-slip velocity condition at all material surfaces and the lateral sidewalls are considered perfectly conducting.

The Lyapunov exponents are computed by measuring trajectory separation in $N_{\lambda}$ independent linearized computations (where $N_{\lambda}$ is the number of desired Lyapunov exponents) $[17,18]$. A driving Rayleigh-Bénard convection system satisfying the Boussinesq equations and subject to appropriate boundary conditions is computed in parallel with the $N_{\lambda}$ linearized equations. The growth of the magnitudes of the perturbation vectors yields the separation in phase-space trajectories from which one can deduce the finite-time Lyapunov exponents [19].

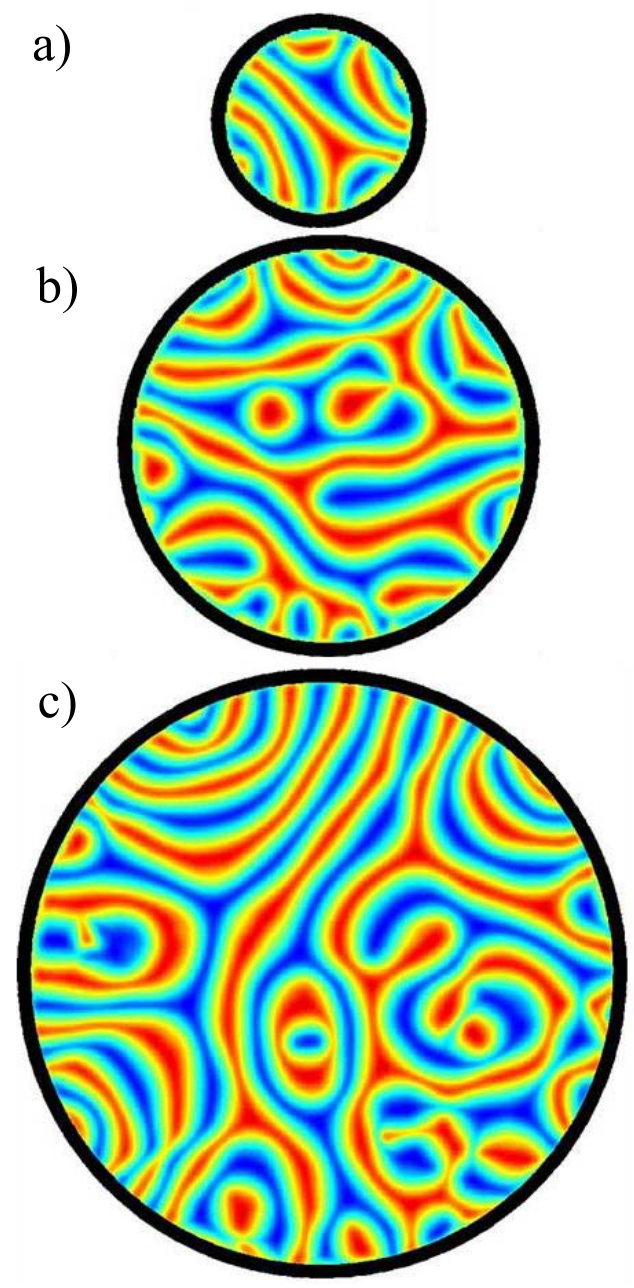

FIG. 1. (Color online) Chaotic flow field from numerical simulations in three different aspect ratio domains $\Gamma=$ (a) 4.72, (b) 10 , and (c) 15. Shown is the two-dimensional temperature field at middepth. Dark regions (red in color) indicate warm rising fluid and lighter regions (blue in color) indicate cool falling fluid. In all simulations $R=6000\left(R / R_{c}=3.5\right.$ where $R_{c}$ is the critical Rayleigh number), $\quad \sigma=1$, with a numerical time step $\Delta t=0.0005$.

Increasing the size of a weakly driven spatially extended system commonly results in spatiotemporal chaos. For example, the pattern dynamics of RBC depend upon the aspect ratio of the convection domain [for a cylindrical domain the aspect ratio $\Gamma=$ (radius) $/($ depth $)]$. This was illustrated experimentally in the early pioneering work of Ahlers and Behringer where heat transport measurements indicated a transition from steady to chaotic dynamics by simply increasing the aspect ratio of the convection layer [20]. For smaller domains the lateral boundaries play a significant role in determining the dynamics and, as the system size increases, there is a transition to bulk-dominated dynamics. In the following we explore spatiotemporal chaos in RBC over a range of system sizes where this transition from boundary- to bulk-dominated dynamics is occurring. In particular, fluid and driving parameters are chosen to correspond to the spiral defect chaos state [21] which consists of the complex timedependent dynamics involving the annihilation and destruc- 

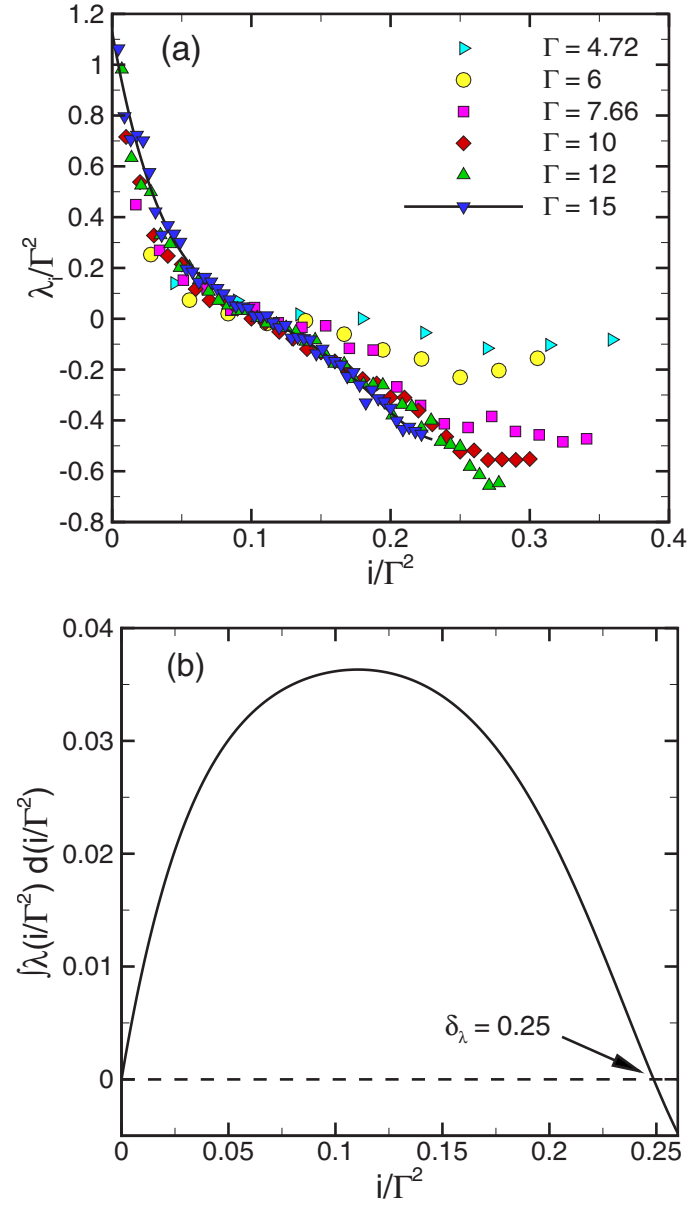

FIG. 2. (Color online) (a) Lyapunov spectrum $\lambda\left(i / \Gamma^{2}\right)$ as a function of $i / \Gamma^{2}$ where $i=1, \ldots, N$ and $N$ is the number of Lyapunov exponents. Results are shown for six different aspect ratios; the solid line is a sixth-order polynomial curve fit to the data for $\Gamma=15$. (b) Calculation of the Lyapunov dimension $\delta_{\lambda}$ as determined by integrating the curve fit for $\Gamma=15$ from (a).

tion of left- and right-handed spiral shaped convection roll structures in large domains [21,22]. Theoretical and experimental investigations of spiral defect chaos in RBC have played an important role in the study of pattern formation. The spiral defect chaos state is only observed in large-aspectratio domains (for cylindrical convection cells $\Gamma \gtrsim 20$ ). For systems smaller than this, as studied here, the lateral walls of the convection domain are important and the dynamics exhibit spatiotemporal chaos that consists of the complicated interactions of many defects including wall foci, dislocations, targets, and roll pinchoff events (see Fig. 1). In this regime, the size of the system significantly affects the dynamics and our study explores spatiotemporal chaos in a finite-sized system which is important for many real-world applications (many naturally occurring systems exhibit complex dynamics with system sizes that cannot be approximated as infinite or periodic). In light of this we perform numerical simulations for the precise conditions of experiment and we study spatiotemporally chaotic RBC over a range of aspect ratios at fixed driving (given by constant $R$ ). Information about the Lyapunov spectra and Lyapunov dimension yield insight into the basic origins and nature of

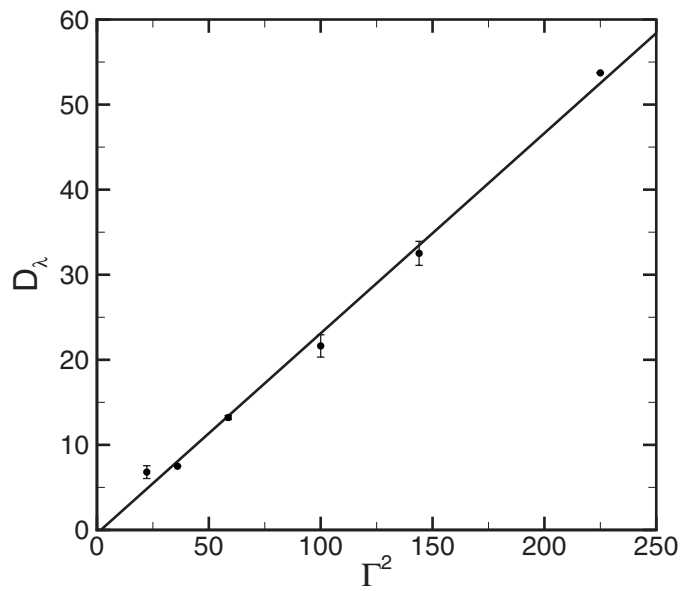

FIG. 3. Extensive chaos in RBC as illustrated by the linear relationship between the Lyapunov dimension $D_{\lambda}$ and system size $\Gamma$. The error bars are determined from multiple simulations for each aspect ratio starting from different random initial conditions for the driving solution. Each data point is the result of two numerical simulations except at $\Gamma=4.72$ and 10 which are for three different initial conditions. If an error bar is not visible it is because the runs yielded nearly identical results for $D_{\lambda}$.

spatiotemporal chaos for an experimentally accessible system.

We have performed a series of large-scale numerical calculations to determine the Lyapunov spectra and Lyapunov dimension of RBC for six aspect ratios spanning $4.72 \leqslant \Gamma \leqslant 15$ with thermal driving given by $R / R_{c}=3.5$ (where $R_{c}=1708$ is the critical Rayleigh number at which convection occurs) and Prandtl number $\sigma=1$. The driving $\mathrm{RBC}$ solution is first initiated from random thermal perturbations and allowed to continue until the initial transients have subsided, at which point it is assumed that the dynamics are on the attractor (an estimate for this time is given by $\Gamma^{2}$ which is the nondimensional time required for heat to diffuse a distance $\Gamma$ ) [23]. At this time, $N_{\lambda}$ independent linearizations are initiated from random initial conditions and these simulations are then allowed to continue at least another $\Gamma^{2}$ time units. In order to keep the magnitude differences resolvable the perturbation vectors are periodically Gram-Schmidt orthonormalized to yield the Lyapunov vectors. For each aspect ratio two or three simulations were performed.

The spectra of Lyapunov exponents $\lambda_{i}$ from these simulations are shown in Fig. 2. For extensive chaos the Lyapunov spectra collapse onto a single curve; our results suggest that this is true for $\Gamma \gtrsim 10$. To indicate this a sixth-order polynomial is fitted to the $\Gamma=15$ data and is shown by the solid line. The deviations of the spectra from this fit for the smalleraspect-ratio domains is the result of boundary-dominated dynamics [19]. The Lyapunov spectrum can be integrated to yield the dimension density $\delta_{\lambda}=D_{\lambda} / \Gamma^{2}$, which is shown in Fig. 2(b) where we find that $\delta_{\lambda} \approx 0.25$. Therefore, for an aspect ratio of 100 (a common size used in experiment) the Lyapunov dimension would be $D_{\lambda}=2500$ indicating the presence of 2500 chaotic degrees of freedom. In the work of Egolf et al. [3] it was found that RBC exhibited extensive chaos in large domains with nonphysical periodic boundary 
conditions. Our work confirms these findings and furthermore indicates that the chaos is also extensive over a range of finite system sizes for experimental conditions.

The extensivity of the chaos is clearly illustrated in Fig. 3 where the Lyapunov dimension is plotted as a function of $\Gamma^{2}$. The slope of the solid line also yields the dimension density. The dimension is extensive for all of the aspect ratios explored except for the smallest system $(\Gamma=4.72)$ where a deviation from extensivity is apparent. An analysis of the flow field dynamics reveals a transition from bulk- to boundarydominated dynamics over the range of system sizes explored [19]. It is interesting to point out that even though the Lyapunov spectra for $\Gamma>4.72$ do not collapse onto the curve fit in Fig. 2 describing the largest aspect ratio $\Gamma=15$ dynamics the Lyapunov spectra still yield Lyapunov dimensions that are extensive.

A volume $\Gamma^{d_{s}}$ contains $D_{\lambda}$ degrees of freedom which suggests a natural length scale for an individual degree of freedom that is given by $\xi=\left(D / \Gamma^{d_{s}}\right)^{-1 / d_{s}}[1]$. For extensive chaos $\xi$ is independent of system size. Our simulations yield a chaotic length scale of $\xi \approx 2$ which suggests that an individual degree of freedom would occupy, on average, an area of $\xi^{2} \approx 4$ (for reference, the width of the convection rolls in Fig. 1 is approximately 1 ). This suggests that localized defect structures could contribute significantly to spatiotemporal dynamics which is corroborated from measurements of the dynamics of the Lyapunov vector fields [3,19].

Our results reveal that $\mathrm{RBC}$ is truly chaotic for experimental conditions and yield a positive leading order Lyapunov exponent. In addition, spatiotemporal chaos in $\mathrm{RBC}$ is extensive over a range of finite system sizes including smaller domains whose dynamics are strongly affected by the presence of lateral sidewalls. The precise manner in which the geometry of the strange attractor changes to maintain extensivity over this range remains an open challenge. However, our results illustrate that with the availability of supercomputers and improved numerical algorithms such fundamental questions can now be addressed quantitatively for the precise conditions of experiment.

We are grateful for many useful interactions with Janet Scheel and Anand Jayaraman. We also acknowledge generous support from Argonne National Laboratory under DOE Contract No. DE-AC02-06CH11357, NSF Teragrid under Grant No. MCA03T028, and Virginia Tech's Terascale Computing Facility for grants of supercomputing resources. The early stages of this research was supported by the U.S. Department of Energy, Grant No. DE-FT02-98ER14892.
[1] M. C. Cross and P. C. Hohenberg, Rev. Mod. Phys. 65, 851 (1993).

[2] L. Sirovich and A. Deane, J. Fluid Mech. 222, 251 (1991).

[3] D. A. Egolf, I. V. Melnikov, W. Pesch, and R. E. Ecke, Nature (London) 404, 733 (2000).

[4] L. Keefe, P. Moin, and J. Kim, J. Fluid Mech. 242, 1 (1992).

[5] J. D. Scheel and M. C. Cross, Phys. Rev. E 74, 066301 (2006).

[6] A. Jayaraman, J. D. Scheel, and H. S. Greenside, Phys. Rev. E 74, 016209 (2006).

[7] I. Goldhirsch, P.-L. Sulem, and S. A. Orszag, Physica D 27, 311 (1987).

[8] J. D. Farmer, E. Ott, and J. A. Yorke, Physica D 7, 153 (1983).

[9] D. Ruelle, Commun. Math. Phys. 87, 287 (1982).

[10] P. Manville, in Macroscopic Modeling of Turbulent Flows, Springer Lecture Notes in Physics, Vol. 230, edited by O. Pironneau (Springer, New York, 1985), pp. 319-326.

[11] H. -W. Xi, R. Toral, nd J. D. Gunton, and M. I. Tribelsky,Phys. Rev. E 53, 3374 (1996).

[12] M. C. Strain and H. S. Greenside, Phys. Rev. Lett. 80, 2306 (1998).
[13] H.-W. Xi, R. Toral, J. D. Gunton, and M. I. Tribelsky, Phys. Rev. E 62, R17 (2000).

[14] S. Tajima and H. S. Greenside, Phys. Rev. E 66, 017205 (2002).

[15] M. P. Fishman and D. A. Egolf, Phys. Rev. Lett. 96, 054103 (2006).

[16] P. F. Fischer, J. Comput. Phys. 133, 84 (1997).

[17] M. R. Paul, K.-H. Chiam, M. C. Cross, P. F. Fischer, and H. S. Greenside, Physica D 184, 114 (2003).

[18] A. Wolf, J. B. Swift, H. L. Swinney, and J. A. Vastano, Physica D 16, 285 (1985).

[19] M. I. Einarsson, Quantifying Spatio-Temporal Chaos in Rayleigh-Bénard Convection (Virgina Polytechnic and State University, Blacksburg, 2006).

[20] G. Ahlers and R. P. Behringer, Phys. Rev. Lett. 40, 712 (1978).

[21] S. W. Morris, E. Bodenschatz, D. S. Cannell, and G. Ahlers, Physica D 97, 164 (1996).

[22] E. Bodenschatz, W. Pesch, and G. Ahlers, Annu. Rev. Fluid Mech. 32, 709 (2000).

[23] M. C. Cross and A. C. Newell, Physica D 10, 299 (1984). 